\title{
Regorafenib, the CORRECT way forward or just another GRIDlock?
}

$\mathrm{S}$ tudies carried out through the past few decades have increased our understanding of the molecular mechanisms behind colorectal cancer (CRC) and gastrointestinal stromal tumours (GIST). The identification of some of the mutations that drive these types of cancer (such as mutations in KIT or PDGFRA in GIST, and mutations in KRAS in CRC) has allowed the development of tyrosine kinase inhibitors (TKI) and antibodies that have provided numerous treatment options improving considerably the clinical outcomes of patients with these malignancies.

Despite these great developments, overall survival for most patients with either metastatic CRC or GIST is still around 3 years. Treatment with the KIT and PDGFR inhibitor imatinib substantially improves the outcome of patients with GIST, but about $80 \%$ of these patients eventually develop resistance to the drug. In turn, sunitinib - another TKI targeting KIT among other kinases- has been proved to be effective in the treatment of imatinibresistant tumours. Unfortunately, the effect lasts for around 1 year, until resistance to this inhibitor also develops.

Things are not much different in the case of CRC, and most tumours progress after treatment with targeted agents, such as bevacizumab, cetuximab, or panitumumab. With this bleak panorama, we cannot help but wonder if there will ever be a targeted therapy that may efficiently manage these cancers. Now, two international phase III trials published in the same issue of The Lancet have assessed whether the oral multikinase inhibitor regorafenib might be the right kinase inhibitor for the job.

In the CORRECT study, the group led by Axel Grothey and Eric Van Cutsem studied the effect of regorafenib versus placebo in the setting of heavily pretreated metastatic CRC. Patients were randomly assigned to receive $160 \mathrm{mg}$ of daily regorafenib $(n=505)$ or placebo $(n=255)$.
The study met its primary end point, as regorafenib was associated with a modest, but statistically significant, improvement in overall survival (6.4 months in the regorafenib group compared with 5.0 months in the placebo group), and also with a significantly improved disease control rate (41\% versus $15 \%$ ).

Although the treatment was not associated with detrimental effects on quality of life, treatment-related toxic events (mainly fatigue and hand-foot skin reaction) occurred in $93 \%$ patients receiving regorafenib compared with $61 \%$ of patients assigned to receive placebo.

In the second trial-the GRID studyGeorge Demetri and colleagues assessed the use of regorafenib versus placebo in the treatment of refractory GIST after treatment failure to at least imatinib and sunitinib. The researchers randomly assigned 133 patients to receive regorafenib (160 mg administered daily) and 66 patients to receive placebo. Regorafenib seemed to be more effective in the setting of refractory GIST than in metastatic CRC. Results from the group led by Demetri showed a marked improvement in progression-free survival, the primary end point of the trial (4.8 months in the regorafenib group and 0.9 months in the placebo group). There was, however, no difference in overall survival, although this is very reasonably explained by the planned study design, which allowed most patients (85\%) in the placebo group to cross-over rapidly to receive regorafenib after disease progression.

As in the CRC scenario, treatmentrelated adverse events (grade 3 or higher) were reported in $98 \%$ patients assigned to the regorafenib group compared with $68 \%$ of patients assigned to receive placebo. The most common regorafenib-related adverse events were hypertension, hand-foot skin reaction and diarrhoea.

So, what's next? For Demetri, this study is just the beginning: "we know

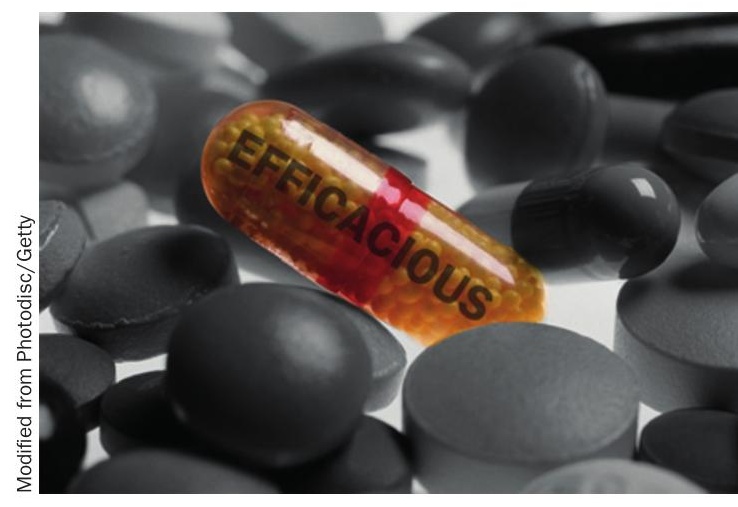

that regorafenib can inhibit many of the mutated proteins and abnormal signals that cause this cancer (GIST), and the next step will be to investigate the molecular mechanisms by which this new treatment can control GIST after resistance develops following other targeted therapies for this aggressive malignancy."

Van Cutsem is also hopeful: “These results should offer hope for a new standard of care in patients with bowel cancer who have no other treatment options left to them. In future, we hope to find subgroups of patients, based on molecular markers, who may experience significantly better survival times on regorafenib and are likely to benefit from it the most."

Certainly, according to the results reported in these two trials, regorafenib seems to be a promising option in the treatment of gastrointestinal tumours.

\section{Teresa Villanueva}

This article originally appeared in Nat. Rev. Clin. Oncol. (doi:10.1038/nrclinonc.2012.222).

\footnotetext{
Original articles Grothey, A. et al. Regorafenib monotherapy for previously treated metastatic colorectal cancer (CORRECT): an international, multicentre, randomised, placebo-controlled, phase 3 trial. Lancet doi:10.1016/ S0140-6736(12)61900-X | Demetri, G. D. et al. Efficacy and safety of regorafenib for advanced gastrointestinal stromal tumours after failure of imatinib and sunitinib (GRID): an international, multicentre, randomised, placebo controlled, phase 3 trial. Lancet doi:10.1016/S01406736(12)61857-1
} 